\title{
Takeuchi technique in the anomalous origin of the left coronary artery from the pulmonary artery
}

\author{
Técnica de Takeuchi en el origen anómalo de la arteria coronaria izquierda de la arteria \\ pulmonara
}

Carlos Domínguez-Massa*, Tomás Heredia-Cambra, Alberto Berbel-Bonillo, Óscar R. Blanco-Herrera, José A. Montero-Argudo, and Fernando Hornero-Sos

Department of Cardiovascular Surgery, Hospital Universitario y Politécnico La Fe, Valencia, Spain

\section{Introduction}

Coronary artery anomalies account for $2.2 \%$ of congenital heart defects ${ }^{1}$, with the most common being anomalous origin of the left coronary artery from the pulmonary artery (anomalous left coronary artery from the pulmonary artery [ALCAPA] $)^{1}$. ALCAPA, or BlandWhite-Garland syndrome, has an incidence of $1 / 30,000-300,000$ newborns ${ }^{1-3}$, with a mortality of $90 \%$ within the $1^{\text {st }}$ year of life ${ }^{2}$. Adult patients are usually asymptomatic or have mitral insufficiency, ischemic heart disease, heart failure, or malignant ventricular arrhythmias (which are the cause of sudden death in $80-90 \%)^{2}$. Most adult patients have significant right coronary artery dilatation due to increased flow to the left coronary artery associated with extensive intercoronary collateral vessels. However, a giant right coronary artery being associated with ALCAPA syndrome is unusual ${ }^{3}$. Mitral regurgitation mechanism in ALCAPA consists of dysfunction of the papillary muscles due to ischemia (usually the posterior papillary muscle), but other times, it is a consequence of mitral annulus dilatation secondary to the spherical configuration adopted by ischemic left ventricle, which causes a coaptation defect of the mitral leaflets ${ }^{1}$.
The diagnosis is made with coronary angiography, but computed tomography (CT) coronary angiography also establishes a clear diagnosis, providing additional anatomical information before surgery ${ }^{4}$. Surgery is recommended in all patients, even if they are asymptomatic'.

\section{Case description}

A 44-year-old woman, who, practicing intense physical exercise, experienced sudden loss of consciousness, with basic cardiopulmonary resuscitation maneuvers starting until she arrived to the emergency medical assistance department. Ventricular fibrillation was documented, which was reversed with electrical defibrillation. The patient was transferred to the hospital. The electrocardiogram showed sinus rhythm, at about $70 \mathrm{bpm}$, with $30^{\circ}$ axis and signs of the left ventricular hypertrophy. Echocardiography showed mild-moderate mitral regurgitation with no other valvular abnormalities. The left ventricle was slightly dilated with hypokinesia of the middle and basal anterior segments and basal area of the septum, with systolic function being preserved. Emergency coronary angiography revealed severe ectasia of the right coronary artery with extensive collateral vessels to the left coronary artery.

\section{Correspondence:}

${ }^{*}$ Carlos Domínguez-Massa 


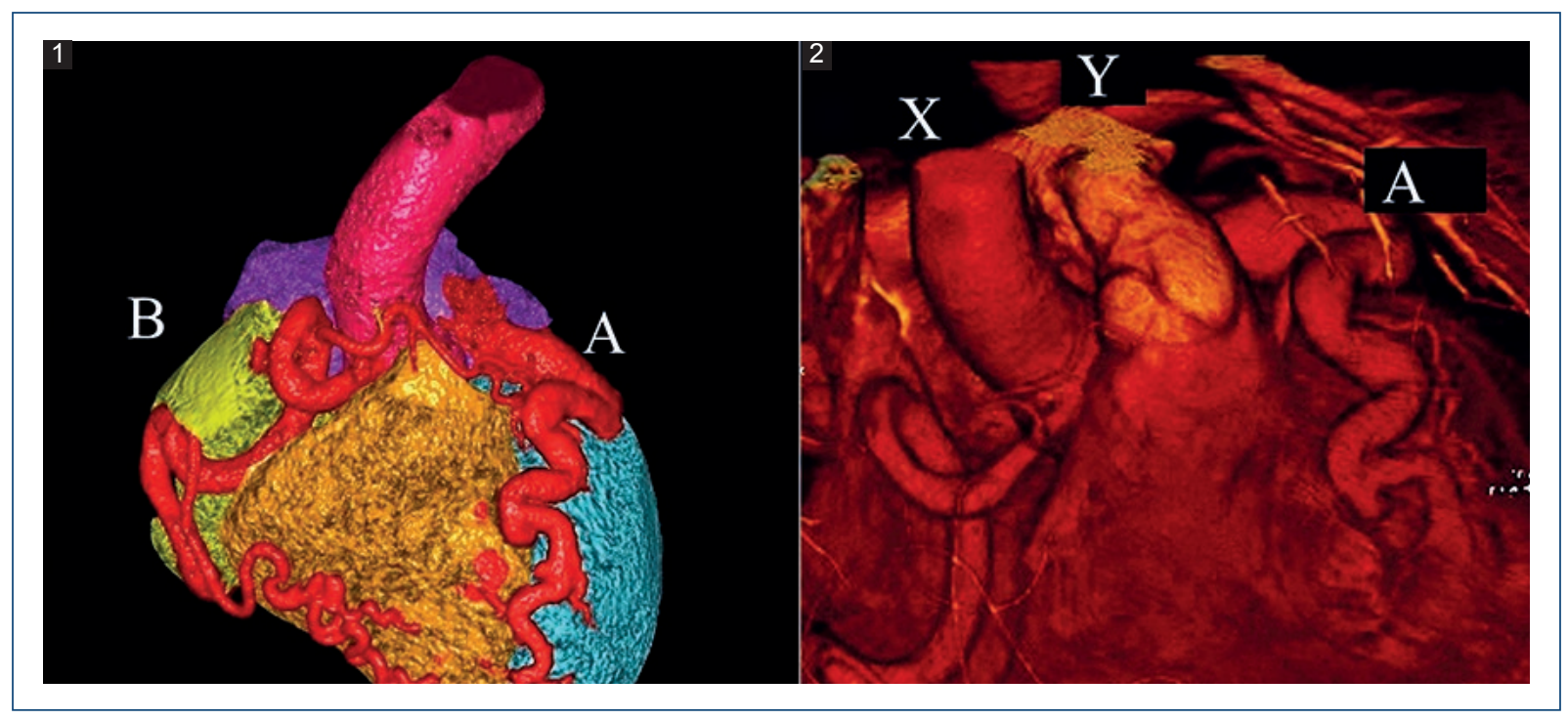

Figure 1. Computed tomography coronary angiography reconstruction. 1: preoperative. The left coronary artery $\mathbf{A}$ : originates in the pulmonary artery. The right coronary artery $\mathbf{B}$ : is ectatic with collateral vessels to the left coronary artery; 2: postoperative. The left coronary artery is tunneled through the pulmonary artery (Y) and to the aorta (X).

When the patient was stabilized, a CT coronary angiography was performed (Fig. 1.1), which was diagnostic of ALCAPA syndrome: the left coronary artery originated at the left lateral level of the pulmonary artery and was filled through a network of collateral vessels dependent on the right coronary artery branches crossing the ventricular myocardium. In addition, there was a significant dilation and tortuosity of all coronary arteries, in associated with the development of collateral vessels in the context of chronic ischemia. A complementary MRI showed ventricular dilation with subendocardial infarction of the anterior, anteroseptal, and anterolateral segments (all viable), associated with moderate mitral regurgitation due to hypokinesia of the anterolateral papillary muscle in relation to chronic ischemia.

The patient was programmed for urgent surgery, which was performed using the Takeuchi technique by means of full median sternotomy. After cardiopulmonary bypass was established, a transverse arteriotomy of the pulmonary artery was performed, with the pulmonary valve and the anomalous origin of the left coronary artery in the pulmonary trunk being visualized, and the birth of the left coronary artery at the left lateral level of the pulmonary artery being appreciated. A pulmonary artery tongue of tissue was obtained at the level of the left coronary artery anomalous origin (Fig. 2A). With a punch, an orifice was made in the aorta and pulmonary artery to create an aortopulmonary window (Fig. 2B).
With the pulmonary artery tissue tongue, a tunnel was formed running from the origin of the left coronary artery to the aortopulmonary window (Fig. 2C). Antegrade cardioplegia was used to check the tightness of the suture. Finally, the pulmonary artery was closed using an autologous pericardium graft to prevent stenosis (Fig. 2D).

The patient was extubated $6 \mathrm{~h}$ after the procedure. As only post-operative complication, she had two episodes of atrial fibrillation with regular hemodynamic tolerance. The first episode was reverted to sinus rhythm with pharmacological cardioversion and the second with electrical cardioversion. In few weeks, a coronary angiography (Fig. 1.2) confirmed the patency of the left coronary artery tunneled through the pulmonary artery. On echocardiography, the left ventricle remained dilated and slight mitral regurgitation persisted. In the examination of the patient at 6-month post-surgery, good clinical evolution was found, with physical exertion progressively being tolerated.

\section{Discussion}

Surgery is recommended in all patients with ALCA$\mathrm{PA}$, even if they are asymptomatic. There are several surgical options, which are divided depending on whether a single coronary system is left or if both coronary systems are restored, with better long-term results ${ }^{1,4}$. The most widely used strategies are direct 

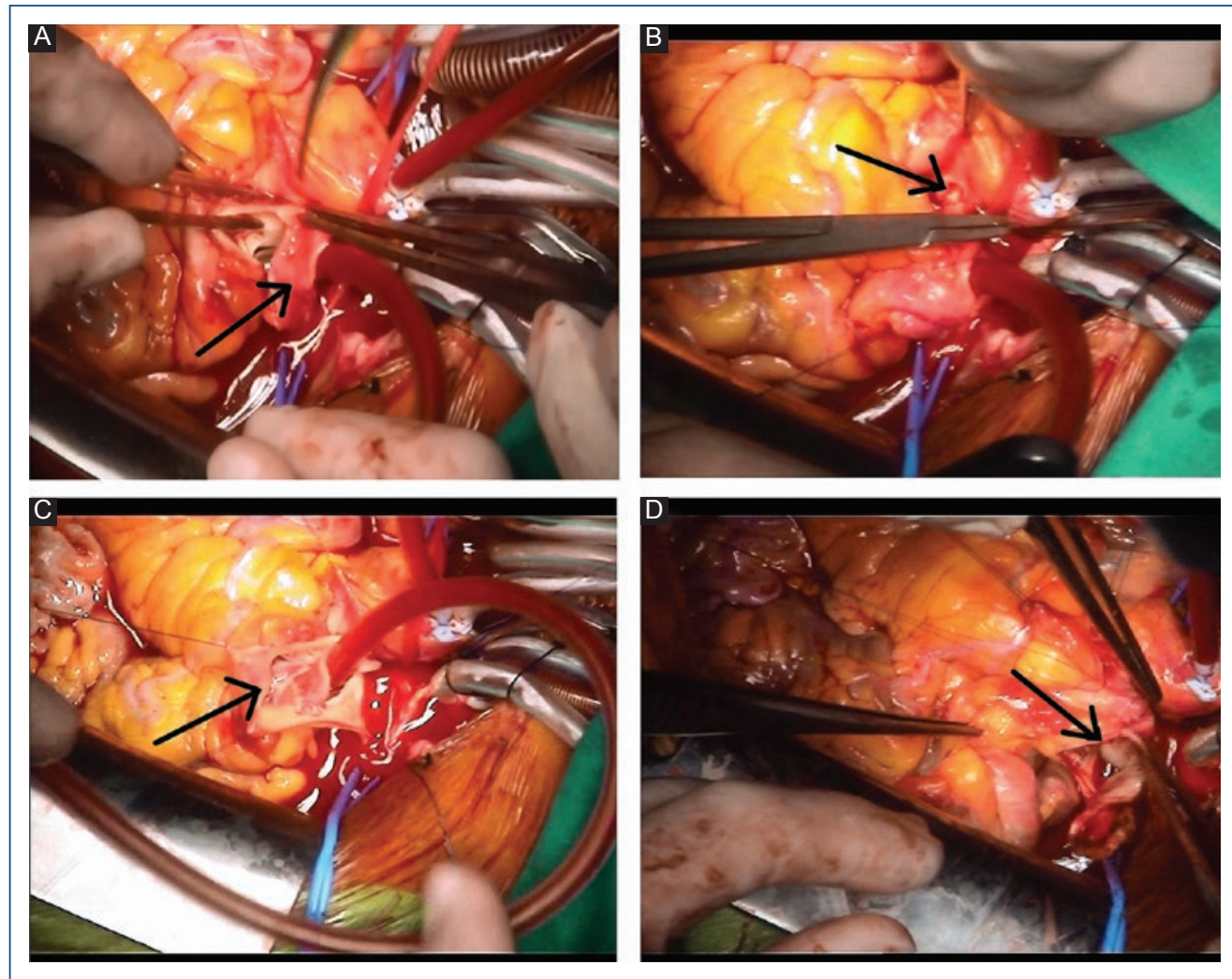

Figure 2. Surgical field photographs. The surgical step explained is marked with an arrow. A: pulmonary arteriotomy to visualize the pulmonary valve and the left coronary artery origin; B: creation of the aortopulmonary window; C: using the pulmonary artery tissue tongue, a left coronary artery tunnel running through the pulmonary artery was formed, for it to originate in the aorta through the aforementioned aortopulmonary window; D: pulmonary artery closure and enlargement with autologous pericardium patch.

reimplantation and the Takeuchi technique ${ }^{5}$. The use of the pulmonary artery in the Takeuchi technique appears to be better than the use of pericardium or other synthetic material since the pericardium entails a higher risk of aneurysm formation due to high pressure, and synthetic materials are more thrombogenic ${ }^{6}$.

The left coronary artery bypass with occlusion or ligation of the anomalous origin is an alternative strategy. However, there are doubts regarding collateral vessels competitive flow, which may affect graft patency, in addition to having the disadvantage of graft occlusion in the medium to long term. Therefore, coronary artery bypass surgery with abnormal coronary artery closure should be reserved for those cases where coronary transfer is not feasible ${ }^{7}$.

Mitral valve surgery in patients with ALCAPA remains controversial. Some authors do not recommend mitral valve surgery during coronary reimplantation, indicating that mitral regurgitation subsequently improves or completely disappears, particularly in childhood. Conversely, other authors recommend performing mitral annuloplasty simultaneously when there is moderate-to-severe mitral regurgitation. The decision to correct mitral regurgitation should be flexible and based on its underlying causes since residual mitral regurgitation has been identified as a risk factor for reoperation, but not for death ${ }^{8,9}$.

\section{Conclusion}

ALCAPA surgery should be individualized in relation to each patient's age, prognosis, and anatomy. The Takeuchi technique is chosen when coronary reimplantation is not possible due to an unfavorable anatomy, as in the presented case, who had an origin at the left lateral level of the pulmonary artery. There is no evidence in literature on the correct attitude toward mitral regurgitation, which usually resolves with the cessation of the triggering ischemic event. 


\section{Ethical disclosures}

Protection of people and animals. The authors declare that no experiments have been carried out on humans or animals for this research.

Confidentiality of data. The authors declare that they have followed the protocols of their work center on the publication of patient data.

Right to privacy and informed consent. The authors declare that no patient data appear in this article.

\section{Funding}

The present investigation has not received specific aid from public or commercial sector agencies or non-profit entities.

\section{Conflicts of interest}

The authors declare that they have no conflicts of interest.

\section{References}

1. Ramírez S, Curi-Curi PJ, Calderón-Colmenero J, García J, Britton C Erdmenger $\mathrm{J}$, et al. Resultados del reimplante coronario para la corrección del origen anómalo de la coronaria izquierda a partir de la arteria pulmonar. Rev Esp Cardiol. 2011;64(8):681-7.

2. Kothari J, Lakhia K, Solanki P, Parmar D, Boraniya H, Patel S. Anomalous origin of the left coronary artery from the pulmonary artery in adulthood: Challenges and outcomes. Korean J Thorac Cardiovasc Surg. 2016:49:383-6.

3. Bajona P, Maselli D, Dore R, Minzioni G. Anomalous origin of the left main artery from the pulmonary artery: Adult presentation with systemic collateral supply and giant right coronary artery aneurysm. J Thorac Cardiovasc Surg. 2007:134:518-20.

4. Joshi SV, Naik AV, Bhalgat PS, Chavan B, Salvi P. An experience with off pump technique for repair of anomalous left coronary artery from pulmonary artery (ALCAPA). Indian Heart J. 2016;68:704-8.

5. Isomatsu Y, Imai Y, Shin'oka T, Aoki M, Iwata Y. Surgical intervention for anomalous origin of the left coronary artery from the pulmonary artery: The Tokyo Experience. J Thorac Cardiovasc Surg. 2001;121:792-7.

6. Kattach $\mathrm{H}$, Anastasiadis $\mathrm{K}$, Jin $\mathrm{XY}$, Pillai R. Two-conduit repair for anomalous origin of the left coronary artery from the pulmonary artery in an adult. J Thorac Cardiovasc Surg. 2004;128:641-2.

7. Rajbanshi BG, Burkhart HM, Schaff HV, Daly RC, Phillips SD, Dearani JA. Surgical strategies for anomalous origin of coronary artery from pulmonary artery in adults. J Thorac Cardiovasc Surg. 2014; 148:220-4.

8. Alexi-Meskishivili V, Nasseri BA, Nordmeyer S, Schmitt B, Weng YG, Böttcher W, et al. Repair of anomalous origin of the left coronary artery from the pulmonary artery in infants and children. J Thorac Cardiovasc Surg. 2011;142:868-74.

9. Pérez-Negueruela C, Arango-Posada C, César S, Bartrons J, Carretero J, Sarquella-Brugada G, et al. Nueve casos de origen anómalo de una arteria coronaria. Cir Cardiov. 2014;21:204-8. 